\title{
Blood clot removal by cryoextraction in critically ill patients with pulmonary hemorrhage
}

\author{
Lars Henning Schmidt ${ }^{1}$, Arik Bernard Schulze ${ }^{1}$, Dennis Goerlich ${ }^{2}$, Christoph Schliemann ${ }^{1}$, Torsten Kessler ${ }^{1}$, \\ Veronika Rottmann $^{1}$, Daniel den Toom ${ }^{1}$, Felix Rosenow ${ }^{3}$, Jan Sackarnd ${ }^{3}$, Georg Evers ${ }^{1 \#}$, Michael Mohr ${ }^{1 \#}$ \\ ${ }^{1}$ Department of Medicine A, Hematology, Oncology and Pneumology, University Hospital Muenster, Muenster, Germany; ${ }^{2}$ Institute of Biostatistics \\ and Clinical Research, Westfaelische Wilhelms-Universitaet Muenster, Muenster, Germany; ${ }^{3}$ Department of Cardiovascular Medicine, Internal \\ Intensive Care Medicine, University Hospital Muenster, Muenster, Germany \\ Contributions: (I) Conception and design: LH Schmidt, G Evers, M Mohr; (II) Administrative support: LH Schmidt, AB Schulze, D Goerlich, C \\ Schliemann, T Kessler, V Rottmann, F Rosenow, J Sackarnd, G Evers, M Mohr; (III) Provision of study materials or patients: LH Schmidt, AB \\ Schulze, C Schliemann, T Kessler, F Rosenow, J Sackarnd, G Evers, M Mohr; (IV) Collection and assembly of data: LH Schmidt, D den Toom, \\ G Evers, M Mohr; (V) Data analysis and interpretation: All authors; (VI) Manuscript writing: All authors; (VII) Final approval of manuscript: All \\ authors. \\ \#These authors contributed equally to this work as shared senior authors. \\ Correspondence to: Lars Henning Schmidt, MD, PD. Department of Medicine A, University Hospital Münster Albert-Schweitzer-Campus 1, 48149 \\ Muenster, Germany. Email: larsHenning.Schmidt@ukmuenster.de.
}

\begin{abstract}
Background: Severe pulmonary hemorrhage is a life-threatening complication in critically ill patients. Due to tracheobronchial obstruction, ventilation is often impaired. Traditionally, rigid bronchoscopy is an option for recanalization. However, in comparison to flexible bronchoscopy, the application of rigid bronchoscopy is more complex. Against this background we evaluated the use of flexible cryo-probes for blood clot removal in critically ill patients.

Methods: Retrospectively, we identified 16 patients (median age: 60 years, 69\% male patients), who suffered from severe airway obstruction due to blood clots. All patients required invasive ventilation and 11 patients depended on extracorporeal membrane oxygenation (ECMO). To remove blood clots, flexible bronchoscopic cryoextraction was performed in $\mathrm{n}=27$ cases, whereas rigid bronchoscopy was only needed in two cases.

Results: Whereas in 9 cases single flexible cryoextraction was successful immediately, the procedure had to be repeated again in 7 patients. In all cases, tracheobronchial obstruction was treated with success and conditions of invasive ventilation were improved. In no case severe complications were observed.

Conclusions: In consideration of the underlying evaluation, we highly recommend flexible cryoextraction as both a safe and less complex technique for blood clot removal in critically ill patients.
\end{abstract}

Keywords: Intensive care medicine; cryo-probe; blood clot removal; extracorporeal membrane oxygenation (ECMO)

Submitted Apr 13, 2019. Accepted for publication Sep 16, 2019.

doi: $10.21037 /$ jtd.2019.09.46

View this article at: http://dx.doi.org/10.21037/jtd.2019.09.46

\section{Introduction}

Often massive pulmonary hemorrhage causes lifethreatening conditions and yields airway obstruction due to blood clots in the tracheobronchial tree (1). With focus on etiology such as lung cancer or tuberculosis, medical procedures like tracheostomy can result in the development of blood clots (2). Therapeutically it is crucial to secure the airways, to correct the bleeding tendency, and to stabilize hemodynamics. Both to stop pulmonary bleeding and to recanalize the airways, interventional bronchoscopy can be 
Table 1 Summary of published flexible cryoextractions for blood clots removal in the tracheobronchial airway system

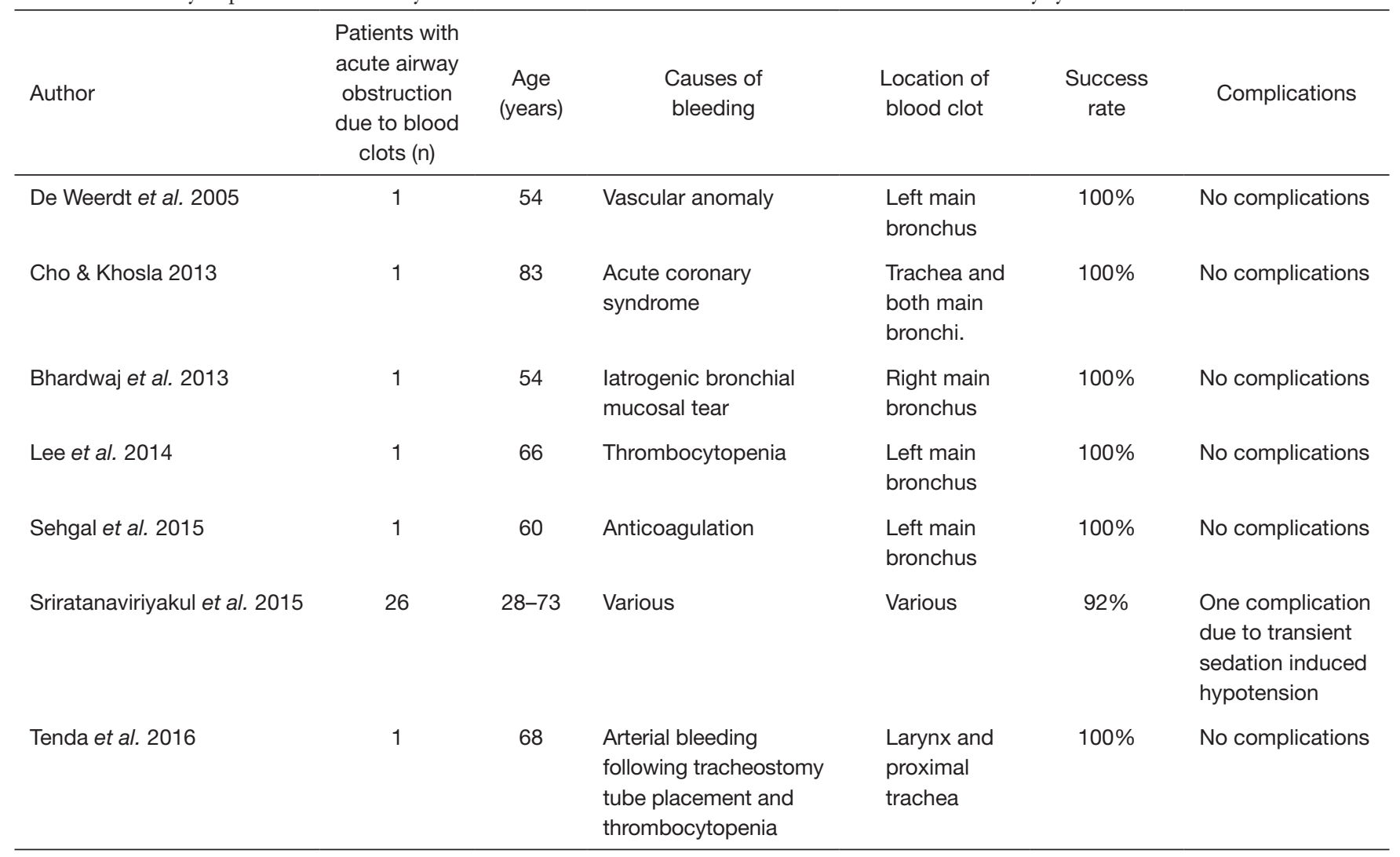

applied $(3,4)$.

Often, critically ill patients with refractory forms of acute respiratory distress syndrome (5-7) under extracorporeal membrane oxygenation (ECMO) (8) suffer from severe pulmonary hemorrhage with obstructing blood clots in the tracheobronchial system. Since ECMO can prevent ventilator-induced lung injuries and thus improve prognosis (9), the application of veno-venous ECMO (vvECMO) has augmented the therapeutic spectrum of intensive care medicine (10). Unfortunately, this development goes along with a higher prevalence of pulmonary hemorrhage. In literature, bleeding complications under ECMO are reported in about $29 \%$ of investigated cases. The estimated mortality rate due to complications however is $6.9 \%$ (11).

In consideration of the life-threatening impact of massive hemorrhage and blood clots, immediate recanalization is urgent. Traditionally rigid bronchoscopy has been used to secure the airway in patients suffering from pulmonary hemorrhage (4). It assures a wide airway and permits the insertion of large instruments or suction catheters (12). However, this rigid technique requires both a specialized team and technical equipment. In contrast, flexible bronchoscopy is less complicated (13). Especially for critically ill patients under mechanical ventilation or patients who require ECMO treatment timesaving therapeutic attempts are crucial. In this context, flexible bronchoscopic cryoextraction, performed bedside on ICU, is an alternative for airway management and especially for the successful removal of blood clots.

Technically, cryo-probe tips are frozen with either nitrous oxide or carbon dioxide. Freezing the blood clots facilitates removal. Successful cryoextraction has been reported in several case reports (Table 1) (14-20). For tumor ablation in symptomatic patients with malignant endobronchial or tracheal tumors the use of cryo-probes is already recommended (21). Since there are fewer studies with focus on the management of blood clots in critically ill patients we evaluated the use of blood clot removal by cryo-probe in critically ill patients, who require ICU treatment. 


\section{Methods}

\section{Study population}

Following the approval of the ethical committee Muenster (Ref. 2016-325-f-S), we evaluated patients, who suffered from blood clots due to pulmonary hemorrhage. Between 2010 and 2017, in total $\mathrm{n}=8,572$ flexible bronchoscopies and 231 rigid bronchoscopies were performed at our University hospital. Among these bronchoscopies we identified $\mathrm{n}=27$ flexible bronchoscopic cryoextractions, which were performed in $\mathrm{n}=16$ patients. In all cases, this procedure always followed unsuccessful bronchoscopic attempts to remove blood clots such as bronchoscopic suction for instance. Of interest, in the same time in total $n=293$ ECMOs were performed using percutaneous cannulation under echocardiography. Data collection was performed retrospectively. Besides clinical data, radiologic data and laboratory parameters (i.e., increased partial thromboplastin time and thrombocyte count) were gathered, too. All laboratory parameters were collected within 48 hours prior first pulmonary bleeding event occurred.

\section{Flexible bronchoscopic cryoextraction}

Flexible bronchoscopy (Olympus Europa SE \& Co. KG, Hamburg, Germany) was carried out bedside using local anesthesia with $2 \%$ lidocaine and under sedation with propofol. In all cases the bronchoscope was introduced via either an endotracheal tube or a tracheostoma on ICU. During bronchoscopy oxygen saturation, blood pressure and ECG were continuously monitored. For cryoextraction, a flexible cryo-probe (ERBE Elektromedizin GmbH, Tuebingen, Germany) with a diameter of either 1.8 or $2.4 \mathrm{~mm}$ was inserted via the working channel. Next, the tip of the cryo probe was placed under bronchoscopic visualization and cooled with nitrous oxide up to $-90^{\circ} \mathrm{C}$ until adhesion was visualized. Following this, both the bronchoscope and the probe were removed together with the adhered blood clot attached to the tip. The procedure was repeated until all blood clots were removed and recanalization was achieved.

\section{Evaluation of prognostic outcome, efficacy and complications}

Since the aim was the recanalization of the central airway system, the procedure was considered as successful if all blood clots were removed completely from the central airways. Next, oxygenation and dependency on mechanical ventilation was studied. Success was defined by visible recanalization after bronchoscopy plus improved radiologic morphology on chest $\mathrm{X}$-ray. Radiologic examination was performed within four hours after flexible cryoextraction.

\section{Statistical analysis}

To describe our study collective, used statistics for mean, median and quartiles as well as frequencies were applied. Overall survival was defined as time (days) between first bronchoscopic cryoextraction and either death or censoring. All statistical tests were performed as exploratory analyses on a local significance level of 0.05 . Since multiplicity adjustment was not carried out, no distinct overall significance level was ascertained. Considering this, our findings might be used to set up new hypotheses. Data collection and calculations were performed using IBM SPSS Statistics ${ }^{\circledR}$ version 25 (released 2017, IBM Corp., Armonk, NY, USA).

\section{Results}

\section{Descriptive analysis of the study population}

The investigated study collective consisted of $n=16$ patients (male: 69\% f female: $31 \%$ ) with various underlying primary diseases. Median age was 60 years. Patients required intensive care treatment due to either complex cardiothoracic surgery ( $\mathrm{n}=8$ cases), acute respiratory distress syndrome (ARDS) ( $\mathrm{n}=6$ cases) or other reasons as pulmonary embolism ( $\mathrm{n}=1$ case) and acute pulmonary hemorrhage of unknown origin ( $\mathrm{n}=1$ case). With focus on the bleeding cause, increased partial thromboplastin time (PTT $\geq 38$ seconds) was found in $\mathrm{n}=4$ cases $(25 \%)$ and both PTT $\geq 38$ seconds plus thrombocytopenia $(<150,000$ thrombocytes $/ \mu \mathrm{L})$ were observed in $\mathrm{n}=11$ cases $(69 \%)$. However, neither thrombocytopenia $(<150,000 / \mu \mathrm{L})$ nor increased PTT (PTT $\geq 38$ seconds) was detected in one case $(6 \%)$. Of interest, $n=11$ patients $(69 \%)$ depended on vvECMO treatment. Descriptive analysis of the study population is summarized in Table 2.

\section{Flexible cryoextraction}

Among $n=8,572$ flexible bronchoscopies we identified $n=27$ flexible bronchoscopic cryoextractions in $\mathrm{n}=16$ patients, who suffered from blood clot obstruction in the tracheobronchial 
Table 2 Baseline characteristics of the investigated study cohort

\begin{tabular}{|c|c|c|}
\hline Characteristics & $\begin{array}{l}\text { Patients } \\
\left(\mathrm{N}_{\text {total }}=16\right)\end{array}$ & $\begin{array}{c}\% \text { of non-missing } \\
\text { variables }\end{array}$ \\
\hline \multicolumn{3}{|l|}{ Age (years) } \\
\hline Median $\left(Q 1^{\star} ; Q^{* *}\right)$ & \multicolumn{2}{|c|}{$60(47.5 ; 68.5)$} \\
\hline \multicolumn{3}{|l|}{ Sex, $N$} \\
\hline \multicolumn{3}{|l|}{ Main diagnoses } \\
\hline \multicolumn{3}{|l|}{ Complex cardiothoracic surgery } \\
\hline Coronary artery bypass surgery plus valve replacement & 2 & 13 \\
\hline Aortic arch replacement & 1 & 6 \\
\hline \multicolumn{3}{|l|}{ Other diagnoses } \\
\hline Acute respiratory distress syndrome (ARDS) with infectious origin & 6 & 38 \\
\hline Pulmonary embolism & 1 & 6 \\
\hline Acute pulmonary hemorrhage of unknown origin & 1 & 6 \\
\hline \multicolumn{3}{|l|}{ Laboratory parameters } \\
\hline Increased partial thromboplastin time ( $\geq 38$ seconds) & 4 & 25 \\
\hline Both thrombocytopenia $(<150,000 / \mu \mathrm{L})$ and increased partial thromboplastin time ( $\geq 38$ seconds) & 11 & 69 \\
\hline Neither thrombocytopenia $(<150,000 / \mu \mathrm{L})$ nor increased partial thromboplastin time ( $\geq 38$ seconds) & 1 & 6 \\
\hline \multicolumn{3}{|l|}{ Extracorporeal membrane oxygenation (ECMO), $\mathrm{N}$} \\
\hline \multicolumn{3}{|l|}{ Survival status, $\mathrm{N}$} \\
\hline Alive & 7 & 44 \\
\hline Dead & 9 & 56 \\
\hline \multicolumn{3}{|l|}{ Causes of death, $\mathrm{N}$} \\
\hline Acute left heart failure & 1 & 11 \\
\hline Multi organ failure & 6 & 67 \\
\hline Massive pulmonary hemorrhage & 1 & 11 \\
\hline Progressive tumor disease & 1 & 11 \\
\hline \multicolumn{3}{|l|}{ Overall survival (days) } \\
\hline Mean $\left(\mathrm{Q} 1^{\star} ; \mathrm{Q} 3^{\star \star}\right)$ & \multicolumn{2}{|c|}{415} \\
\hline Median (minimum; maximum) & \multicolumn{2}{|c|}{$23(7 ; 827)$} \\
\hline
\end{tabular}

${ }^{\star} \mathrm{Q} 1$, stands for first quartile; ${ }^{\star *} \mathrm{Q}$, stands for third quartile. 


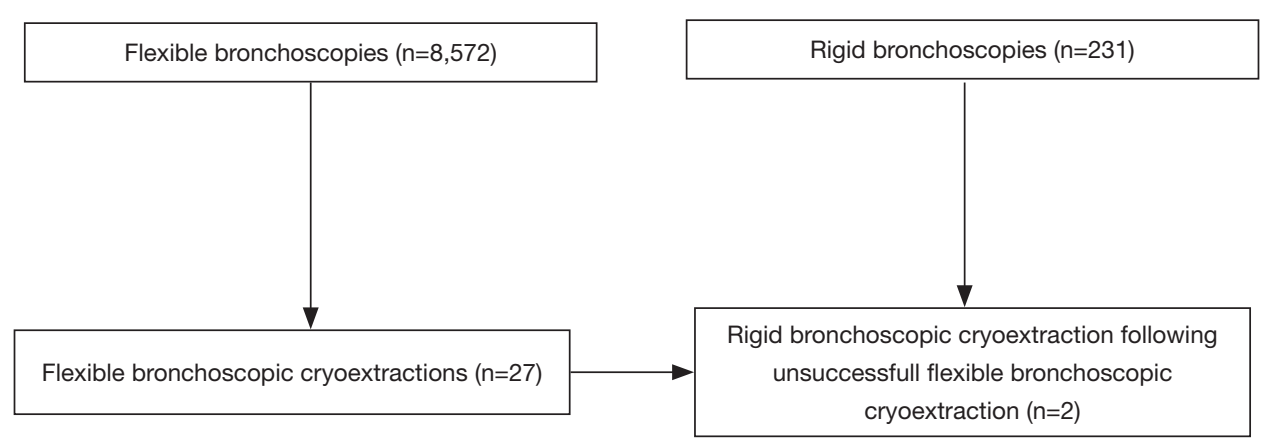

Figure 1 Selection of the investigated study collective is demonstrated in this flow diagram.

Table 3 Procedural information of flexible cryoextraction in the investigated study collective

\begin{tabular}{|c|c|c|}
\hline Variable & Flexible cryoextractions $(n=27)$ & $\%$ of non-missing variables \\
\hline Trachea plus both main bronchi & 10 & 37 \\
\hline Left main bronchus & 6 & 22 \\
\hline Left lower lobe bronchus & 3 & 11 \\
\hline Right lower lobe bronchus & 4 & 15 \\
\hline Right main bronchus & 1 & 4 \\
\hline \multicolumn{3}{|l|}{ Bleeding following cryoextraction, $\mathrm{N}$} \\
\hline No bleeding following cryoextraction & 24 & 89 \\
\hline \multicolumn{3}{|c|}{ Complications within 48 hours following cryoextraction, $\mathrm{N}$} \\
\hline No complications & 27 & 100 \\
\hline Complications & 0 & 0 \\
\hline
\end{tabular}

APC, argon plasma coagulation.

airways. In $n=9$ patients one cryoextraction was sufficient. In $n=4$ patients two cryoextractions, in $n=2$ patients three cryoextractions and in one patient even four cryoextractions had to be performed. To stop further bleeding following cryoextraction, argon plasma coagulation (APC) was applied additionally in one case. In another case bronchial artery embolization was initiated to stop both bleeding and blood clot formation. Since in two patients airway recanalization was not feasible using flexible cryo-probes, rigid bronchoscopy with rigid forceps was done. Of interest, all procedures were performed bedside under invasive ventilation on our intensive care units. The selection process is demonstrated in a flow diagram (Figure 1).

With focus on the location, in ten cases trachea plus both main bronchi were obstructed due to blood clots. In 9 cases, left bronchial system (left main bronchus: $\mathrm{n}=6$ cases; left lower lobe bronchus: $\mathrm{n}=3$ cases) and in eight cases the right bronchial system (right main bronchus: $\mathrm{n}=1$ case; right lower lobe bronchus: $\mathrm{n}=4$ cases; bronchus intermedius: $\mathrm{n}=3$ cases) were blocked (Table 3).

\section{Therapeutic effects, complications and prognostic outcome}

A representative example of cryoextraction is given in 


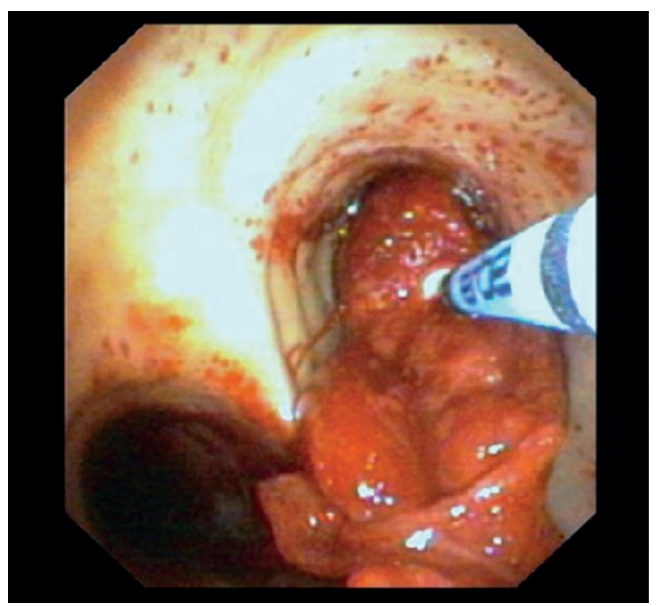

Figure 2 Representative extraction of blood clot from the right main bronchus.

Figure 2. As defined by both visible recanalization and improved radiologic morphology on chest X-ray following bronchoscopic cryoextraction, less atelectasis was observed in $\mathrm{n}=15$ patients $(94 \%)$. Whereas in 9 cases single application was successful immediately after bronchoscopic cryoextraction, the procedure had to be repeated again in 7 patients (Table 4). Even though in one patient (6\%) both APC and cryoextraction was performed repetitively for four times, massive pulmonary hemorrhage could not be controlled. This patient died within six days following the first bronchoscopic cryoextraction. In total $n=9$ patients $(56 \%)$ died in the course of ICU treatment. With focus on the cause of death, we identified multi-organ failure in $\mathrm{n}=6$ cases, massive pulmonary hemorrhage in $\mathrm{n}=1$ case, acute left heart failure in $n=1$ case and progressive tumor disease in $n=1$ case. Median overall survival after first flexible bronchoscopic cryoextraction was 23 days. Of interest, no treatment related complications were observed following flexible cryoextraction in the full study collective. Besides, overall survival of patients, who depended on ECMO did not differ significantly from patients without ECMO treatment $(\mathrm{P}=0.726$, logrank test, Figure 3$)$.

\section{Discussion}

In the clinical course of critically ill patients on ICU, airway obstruction due to blood clots following pulmonary hemorrhage is not a rare phenomenon. With regard to the etiology, there is a huge variety of underlying causes such as trauma, cardiothoracic surgery, pulmonary infections, tumors, pulmonary infarcts or ECMO treatment. Depending on the extent of obstruction, blood clot associated airway obstruction can yield ventilatory impairment and thus it is a life-threatening event. Usually, tracheobronchial obstruction due to massive blood clots in the airways presents with either dystelectasis or even atelectasis upon radiologic examination. Consequently, the next diagnostic step in clinical practice is to rule out tracheobronchial obstruction by immediate flexible bronchoscopic inspection. Therapeutically, in some cases suctioning or forceps extraction via flexible bronchoscopes might solve the obstruction. However, if initial therapy is not successful further techniques are required. In the past rigid bronchoscopy has been applied as the next therapeutic step for airway management $(2,4,22,23)$. Alternatively, bronchoscopic cryo-probes can serve as a further therapeutic option for the extraction of obstructing blood clots. For malignant tracheobronchial obstruction the use of cryotherapy is already accepted (24). Here cryoprobes are applied as an inexpensive but also safe technique, which can easily be performed in an endoscopy suite (25) or even bedside on ICU. Whether flexible cryoextraction for blood clot removal is superior in comparison to rigid bronchoscopy is not clarified, yet. To our knowledge, there are no prospective systematic studies comparing both techniques to each other. Against this background, we evaluated 16 critically ill patients who suffered from airway obstruction due to blood clots. Since some patients required more than just one cryoextraction, in total 27 flexible cryoextractions were performed to remove blood clots.

Our study collective contains patients of all ages between 29 and 77 years, similar to other published studies (14-20). All identified patients depended on mechanical ventilation. Additionally, almost two third of the investigated patients needed vvECMO treatment due to a variety of underlying primary diagnoses such as ARDS or following complex cardiothoracic surgeries. Whereas in 25 procedures flexible cryoextraction was successful, in two cases there was the need to switch to rigid bronchoscopy since airway recanalization was not feasible using flexible cryoextraction. The success rate of $93 \%$ is high and corresponds well to other published data (14-20). Of interest, no relevant difference with regard to univariate overall survival was observed between patients, who depended on ECMO and patients without ECMO treatment.

Focusing on practicability, even distant clots in the lower lobe bronchi were reached with the tip of the cryoprobe, if inserted via a flexible bronchoscope. Besides, 
Table 4 Bronchoscopic findings and results on chest X-ray following flexible cryoextraction

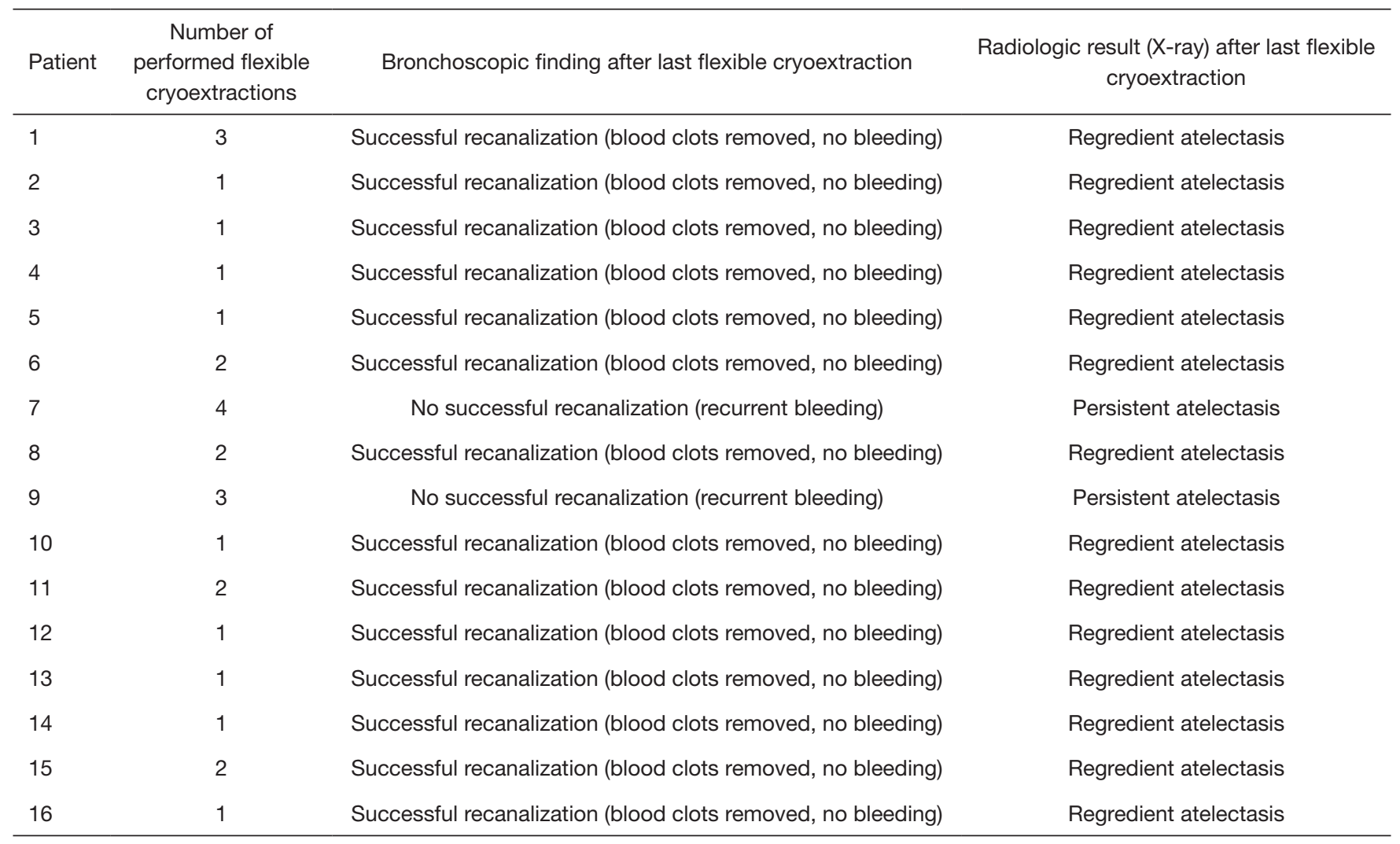

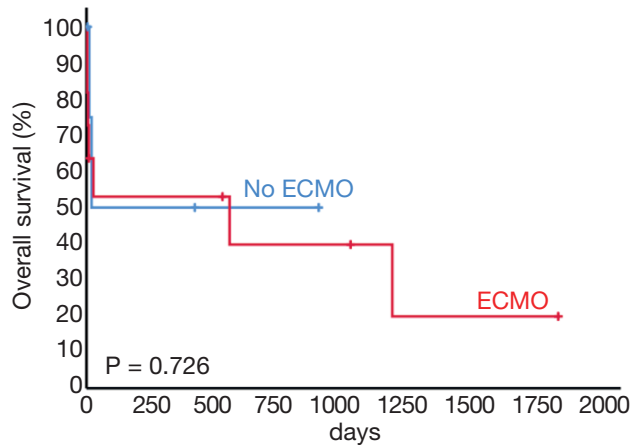

Figure 3 Prognostic impact of ECMO treatment on overall survival in patients who were treated with cryoextraction to treat blood clot obstruction in the tracheobronchial system. ECMO, extracorporeal membrane oxygenation.

repetitive application is feasible and in our study it was conducted in 7 patients. In one patient cryoextraction was repeated for four times. Unfortunately, this patient died due to severe pulmonary hemorrhage, even though APC was applied. With regard to an increased risk of repetitive cryoextractions and in consideration of its impact on the healing process (19), we desisted from a fifth cryoextraction in this case. Without doubt, critical weighting of risk and benefits always needs further consideration, before this therapeutic step is chosen. There is a potential risk of perforating the tracheobronchial system and damaging the bronchoscope's working channel (19), too. Further risks are pulmonary hemorrhage and massive bleeding following successful removal of blood clots in patients with impaired blood coagulation or thrombocytopenia. Moreover, using the existing endobronchial tube can also be problematic, if removed blood clots yielded mechanical obstruction in the tube.

According to our evaluation, none of the investigated patients suffered from any complications associated with this therapy. The low complication rate is also supported by other study groups (14-20). From our perspective, technical performance of flexible cryoextraction in general is less complicated and better feasible in both sedated and intubated patients in comparison to awake, dyspneic and hypoxemic patients. 
In consideration of the bigger diameter, which is offered by rigid bronchoscopy, it is still the gold standard for blood clot removal. However, rigid bronchoscopy is the more complex technique and requires both more technical equipment and human resources. Against this background, flexible cryoextraction can be advantageous in distinct acute situations, especially when time is a crucial factor. Moreover, more distal sites can be reached with the flexible bronchoscope while using the cryo-probe. Even though, flexible cryoextraction is a bedside technique, all patients, who require blood clot removal due to pulmonary bleeding are critical patients and require both intensive care and treatment. If possible, a surgical team should be available as backup. In this context conversion to rigid bronchoscopies might increase the level of stress for ICU patients with potential impact on patients' safety (19). Since all presented patients were critically ill and required ICU treatment, our data speak for the application of flexible cryoextraction as a safe and effective tool for blood clot removal. Due to the freezing process, this technique facilitates en bloc removal of blood clots and thus yields rapid airway recanalization. Even though, the time period of the procedure was not evaluated in this study, according to our experience, the whole procedure usually lasts less than ten minutes until complete recanalization. Potentially, there is also a positive effect on bleeding due to vasoconstriction and hemostatic effects following cryotherapy (26).

Major limitations of our study are due to the limited number of investigated patients and its retrospective singlecenter design. However, in consideration of the investigated critically ill patients and from an ethic point of view it is difficult to conduct prospective head-to-head comparisons for both techniques.

\section{Conclusions}

According to our data, we recommend the use of flexible bronchoscopic cryoextraction as both a safe and effective technique for blood clot removal in critically ill patients who require ICU treatment. This includes patients who depend on ECMO, too. Since this procedure can easily be performed bedside via an endotracheal tube, it might reduce the need for more invasive approaches such as rigid bronchoscopy and thus it contributes to patient's safety.

\section{Acknowledgments}

None.

\section{Footnote}

Conflicts of Interest: The authors have no conflicts of interest to declare.

Ethical Statement: The authors are accountable for all aspects of the work in ensuring that questions related to the accuracy or integrity of any part of the work are appropriately investigated and resolved. The study was approved by the ethical committee Muenster (Ref. 2016325-f-S).

\section{References}

1. Ernst A, Feller-Kopman D, Becker HD, et al. Central airway obstruction. Am J Respir Crit Care Med 2004;169:1278-97.

2. Arney KL, Judson MA, Sahn SA. Airway obstruction arising from blood clot: three reports and a review of the literature, Chest 1999;115:293-300.

3. Radchenko C, Alraiyes AH, Shojaee S. A systematic approach to the management of massive hemoptysis. J Thorac Dis 2017;9:S1069-86.

4. Yendamuri S. Massive airway hemorrhage. Thorac Surg Clin 2015;25:255-60.

5. Ranieri VM, Rubenfeld GD, Thompson BT, et al. Acute respiratory distress syndrome: the Berlin Definition. JAMA 2012;307:2526-33.

6. Villar J, Pérez-Méndez L, Blanco J, et al. A universal definition of ARDS: the $\mathrm{PaO} / \mathrm{FiO} 2$ ratio under a standard ventilatory setting - a prospective, multicenter validation study. Intensive Care Med 2013;39:583-92.

7. Bellani G, Laffey JG, Pham T, et al. Epidemiology, patterns of care, and mortality for patients with acute respiratory distress syndrome in intensive care units in 50 countries. JAMA 2016;315:788-800.

8. Del Sorbo L, Cypel M, Fan E. Extracorporeal life support for adults with severe acute respiratory failure. Lancet Respir Med 2014;2:154-64.

9. Terragni PP, Rosboch G, Tealdi A, et al. Tidal hyperinflation during low tidal volume ventilation in acute respiratory distress syndrome. Am J Respir Crit Care Med 2007;175:160-6.

10. Paden ML, Conrad SA, Rycus PT, et al. Extracorporeal life support organization registry report 2012. ASAIO J 2013;59:202-10.

11. Vaquer S, de Haro C, Peruga P, et al. Systematic review and meta-analysis of complications and mortality of 
veno-venous extracorporeal membrane oxygenation for refractory acute respiratory distress syndrome. Ann Intensive Care 2017;7:51.

12. Parrish S, Krimsky W, Browning R, et al. Novel approaches to the patient with massive hemoptysis. J Community Hosp Intern Med Perspect 2012. doi: 10.3402/jchimp.v2i1.14784.

13. Gupta AA, Sehgal IS, Dhooria S, et al. Indications for performing flexible bronchoscopy: Trends over 34 years at a tertiary care hospital. Lung India 2015;32:211-5.

14. De Weerdt S, Noppen M, Remels L, et al. Successful removal of a massive endobronchial blood clot by means of cryotherapy, J Bronchol Interv Pulmonol 2005;12:23-4.

15. Cho AH, Khosla R. A case of massive airway clotting after use of activated factor VII for massive hemoptysis: management with flexible bronchoscopy and cryoadhesion. J Bronchology Interv Pulmonol 2013;20:276-77.

16. Bhardwaj B, Bhardwaj H, Youness HA, et al. Bronchoscopic cryoextraction: a novel approach for the removal of massive endobronchial blood clots causing acute airway obstruction, Southwest J Pulm. Crit Care 2013;7:184-9.

17. Lee H, Leem CS, Lee JH, et al. Successful removal of endobronchial blood clots using bronchoscopic cryotherapy at bedside in the intensive care unit. Tuberc Respir Dis (Seoul) 2014;77:193-6.

18. Sehgal IS, Dhooria S, Agarwal R, et al. Use of a Flexible Cryoprobe for Removal of Tracheobronchial Blood Clots.
Respir Care 2015;60:e128-31.

19. Sriratanaviriyakul N, Lam F, Morrissey BM, et al. Safety and Clinical Utility of Flexible Bronchoscopic Cryoextraction in Patients With Non-neoplasm Tracheobronchial Obstruction: A Retrospective Chart Review. J Bronchology Interv Pulmonol 2015;22:288-93.

20. Tenda ED, Yakub A, Pitoyo CW, et al. Combination of bronchoscopic cryoextraction and argon plasma coagulation in treatment of total central airway obstruction caused by giant blood clot formation in massive airway bleeding. Respir Med Case Rep 2016;19:9-11.

21. Schumann C, Hetzel M, Babiak AJ, et al. Endobronchial tumor debulking with a flexible cryoprobe for immediate treatment of malignant stenosis. J Thorac Cardiovasc Surg. 2010;139:997-1000.

22. Alraiyes AH, Machuzak MS. Rigid bronchoscopy. Semin Respir Crit Care Med 2014;35:671-80.

23. Batra H, Yarmus L. Indications and complications of rigid bronchoscopy. Expert Rev Respir Med 2018;12:509-20.

24. Harris K, Puchalski J, Sterman D. Recent Advances in Bronchoscopic Treatment of Peripheral Lung Cancers. Chest 2017;151:674-85.

25. Mathur PN, Wolf KM, Busk MF, et al. Fiberoptic bronchoscopic cryotherapy in the management of tracheobronchial obstruction. Chest 1996;110:718-23.

26. Saladi L, Lvovsky D. Organized blood clot masquerading as endobronchial tumor: A review of management and recent advances. Respir Med Case Rep 2018;24:165-9.
Cite this article as: Schmidt $\mathrm{LH}$, Schulze AB, Goerlich D, Schliemann C, Kessler T, Rottmann V, den Toom D, Rosenow F, Sackarnd J, Evers G, Mohr M. Blood clot removal by cryoextraction in critically ill patients with pulmonary hemorrhage. J Thorac Dis 2019;11(10):4319-4327. doi: 10.21037/ jtd.2019.09.46 